\title{
BMJ Open Effect of an interactive therapeutic robotic animal on engagement, mood states, agitation and psychotropic drug use in people with dementia: a cluster-randomised controlled trial protocol
}

\author{
Wendy Moyle, ${ }^{1,2,3}$ Elizabeth Beattie, ${ }^{3}$ Brian Draper, ${ }^{4}$ David Shum, ${ }^{1,5}$ \\ Lukman Thalib, ${ }^{6}$ Cindy Jones, ${ }^{1,2}$ Siobhan O'Dwyer, ${ }^{1,2,3}$ Cindy Mervin ${ }^{1,7}$
}

To cite: Moyle W, Beattie E, Draper B, et al. Effect of an interactive therapeutic robotic animal on engagement, mood states, agitation and psychotropic drug use in people with dementia: a cluster-randomised controlled trial protocol. BMJ Open 2015;5:e009097. doi:10.1136/bmjopen-2015009097

- Prepublication history for this paper is available online. To view these files please visit the journal online (http://dx.doi.org/10.1136/ bmjopen-2015-009097).

Received 16 June 2015 Revised 16 July 2015 Accepted 23 July 2015

CrossMark

For numbered affiliations see end of article.

Correspondence to Professor Wendy Moyle; w.moyle@griffith.edu.au

\section{ABSTRACT}

Introduction: Apathy, agitated behaviours, Ioneliness and depression are common consequences of dementia. This trial aims to evaluate the effect of a robotic animal on behavioural and psychological symptoms of dementia in people with dementia living in long-term aged care.

Methods and analysis: A cluster-randomised controlled trial with three treatment groups: PARO (robotic animal), Plush-Toy (non-robotic PARO) or Usual Care (Control). The nursing home sites are Australian Government approved and accredited facilities of 60 or more beds. The sites are located in South-East Queensland, Australia. A sample of 380 adults with a diagnosis of dementia, aged 60 years or older living in one of the participating facilities will be recruited. The intervention consists of three individual 15 min non-facilitated sessions with PARO or PlushToy per week, for a period of 10 weeks. The primary outcomes of interest are improvement in agitation, mood states and engagement. Secondary outcomes include sleep duration, step count, change in psychotropic medication use, change in treatment costs, and staff and family perceptions of PARO or Plush-Toy. Video data will be analysed using Noldus XT Pocket Observer; descriptive statistics will be used for participants' demographics and outcome measures; cluster and individual level analyses to test all hypotheses and Generalised Linear Models for cluster level and Generalised Estimation Equations and/or Multi-level Modeling for individual level data.

Ethics and dissemination: The study participants or their proxy will provide written informed consent. The Griffith University Human Research Ethics Committee has approved the study (NRS/03/14/HREC). The results of the study will provide evidence of the efficacy of a robotic animal as a psychosocial treatment for the behavioural and psychological symptoms of dementia. Findings will be presented at local and international conference meetings and published in peer-reviewed journals.

\section{Strengths and limitations of this study}

- The study proposes the assessment of a novel technology that people with dementia can interact with.

- The strengths of the study include its relatively large sample size, three treatment groups, longterm sustainability follow-up, video and physiological data and comparative cost analysis.

- Limitations include the intervention being conducted only during the afternoon rather than at the onset of agitation, and no comparison between a live animal and robot animal.

Trial registration number: Australian and New Zealand Clinical Trials Registry number ACTRN12614000508673 date registered 13/05/2014.

\section{INTRODUCTION}

Background

Approximately 35.6 million people globally and 330000 Australians $^{1}$ have dementia. With the ageing of the population, this number is expected to double every 20 years. $^{2}$ Dementia is one of the major reasons for admission into long-term aged care. People with dementia may present with agitated behaviours that cause stress for the person him/herself, those who care for them, and other residents in care facilities. Apathy, loneliness and depression also commonly occur in people with dementia and can make it challenging for care staff to engage them in meaningful activities, which in turn places them at high risk for further 
cognitive and functional decline. ${ }^{3}$ Over $50 \%$ of all residents with dementia in Australia are reported to have behaviours such as physical aggression, agitation, vocal disruption and chronic mood disturbance. ${ }^{1}$ Many of the behavioural and psychological symptoms of dementia, such as agitation, can lead to staff stress and burnout, ${ }^{4}$ and can also result in negative staff attitudes and reduced empathy. ${ }^{5}$ Consequently, feelings of frustration and agitation are heightened, which may lead to the additional regular use of antipsychotic medication. ${ }^{1}$ Serious adverse drug effects include increase risk of stroke, sedation, confusion, falls and mortality in addition to the high cost of pharmaceutical treatment and overuse. ${ }^{6}$ This project aims to assess the impact of a robotic animal called PARO on agitation, mood states, and engagement, sleep duration, step count, change in psychotropic medication use, change in treatment costs and staff and family perceptions of PARO or Plush-Toy.

\section{Rationale for the proposed trial}

Robotic pets, also called emotional or therapeutic robots, have recently been introduced as companions for people with cognitive impairment or/and physical problems. ${ }^{7-9}$ The advantages of robotic pets have been listed as: its highly imitative, life-like behaviour; the modelling of emotional states usually experienced by humans; and the provision of alternative models of communication (eg, tactile-kinesthetic, visual sensory, emotional and social). In addition an interaction can occur between a resident and robotic animal without the need for a carer to be present (ie, robotic pets can fill those inevitable gaps in the day when the resident is alone because the carer is otherwise occupied). Descriptive studies have reported improvements in relationships and loneliness, relaxation and motivation, and socialisation in older people who have interacted with pet-type robots. ${ }^{7-9}$ Previous research in a Japanese aged care facility found that PARO, a harp seal robot, increased residents' social interaction and decreased stress. ${ }^{10}$ These international studies, however, are limited by serious methodological problems including small sample sizes (or single-case studies), no control groups, limited outcome measures, and studies including both people with and without dementia. It remains unclear whether therapeutic robots such as PARO have more benefit compared with non-robotic interventions or usual care for dementia related problems such as agitation, reduced mood and lack of engagement. The current study seeks to extend the existing evidence about robotic animal therapy. It will build on the findings of our pilot study ${ }^{11}$ and provide evidence to determine whether PARO is, indeed, a short-term, low-risk, non-pharmacological intervention that produces tangible, positive psychological outcomes for people with dementia.

\section{AIMS}

This cluster-randomised controlled trial (C-RCT), in which long-term care (LTC) facilities will be randomised to one of three treatment groups (PARO, Plush Toy or Usual Care (UC)), aims to:

1. Compare UC with an innovative interactive therapeutic robot (PARO) and a look-alike Plush Toy (PT -A PARO with the artificial intelligence and robotic aspects disabled) in reducing agitation, improving mood states and engagement; as well as improving physical activity and sleep duration.

2. Evaluate the acceptability of PARO and Plush Toy for staff and family.

3. Conduct a comparative cost analysis of PARO and Plush-Toy with UC as non-pharmacological treatment alternatives to manage and reduce agitation, and improve mood states and engagement in people with dementia.

\section{METHODS}

Study setting

This study will be a multisite trial in 35 Australian Government approved and accredited LTC facilities of 60 or more beds in South East Queensland, Australia, within a $100 \mathrm{~km}$ radius of the Brisbane central business district. All participants will be living in one of the participating facilities.

\section{Trial design}

The trial is a C-RCT. A C-RCT eliminates the potential for contamination in a simple RCT where residents in the same facility are allocated to different groups and participants in the control group might be inadvertently exposed to the intervention due to the nature and layout of care facilities. Because PARO moves and makes noise, a C-RCT will also help to ensure blinding (for participants, family and staff members) is not compromised.

\section{Eligibility criteria}

We will recruit 380 residents, aged 60 years or older from the 35 participating facilities with a range of demographic profiles, a documented diagnosis of dementia (any type) or severity; and a Rowland Universal Dementia Assessment Scale (RUDAS) ${ }^{12}$ score of 22 or less (to accommodate Culturally and Linguistically Diverse People).

We will exclude residents who are in respite care or have: a dual diagnosis of a serious or persistent mental illness (eg, schizophrenia); a terminal illness; or experiencing unremitting pain or distressing physical symptoms. Simultaneous use of PARO or PT will not be permitted during the trial.

\section{Study intervention}

PARO

Participants at sites allocated to the PARO treatment group will receive three individual non-facilitated $15 \mathrm{~min}$ 
PARO sessions per week (Mon, Wed, Fri), between the hours of 1300-1700, for a period of 10 weeks. PARO, developed by Dr Shibata, a researcher at Japan's National Institute of Advanced Industrial Science and Technology, is a therapeutic pet-type robot, with the appearance of a baby harp seal and about the size of a newborn baby. It has tactile sensors and moves its tail and flippers, and opens its eyes when petted. Artificial intelligence software changes the robot's behaviours based on an array of sensors that monitor sound, light, temperature and touch. It responds to sounds, can learn its name and learns to respond to frequent words used by its owner. It can show emotions such as surprise, happiness, and anger, and will cry if it is not receiving sufficient attention. It produces sounds similar to a real baby seal and is active during the day and asleep at night. Participants will be given the PARO and the research assistant (RA) will repeat a short script: "This is PARO. PARO is a companion animal. PARO can move. PARO will respond when you touch and stroke PARO and to your voice when you speak to PARO. Look at the reaction of PARO'S eyes. I will leave you with PARO for a short time for you to get to know PARO. I will sit over there and read my book while you have PARO."

\section{Plush-Toy}

Participants at sites allocated to the PT treatment group will receive three individual non-facilitated sessions (with PT) per week (Mon, Wed, Fri), for 15 min per session, between the hours of 1300-1700, for a period of 10 weeks. The PT is a PARO that has had all robotic and artificial intelligence capabilities disabled. Participants will be given the PT and the RA will repeat a short script: "This is $\mathrm{X}$ (PT name). $\mathrm{X}$ is a companion animal. $\mathrm{X}$ cannot move. $\mathrm{X}$ can be touched and stroked and spoken to. Look at X's eyes. I will leave you with X for a short time for you to get to know X. I will sit over there and read my book while you have X."

RAs receive at least $5 \mathrm{~h}$ of training specific to the intervention. These trained RAs will deliver and introduce the PARO or PT to participants at the start of each session and collect the PARO or PT from participants at the end of each session. In the session, residents will be left alone to interact with PARO or PT, however, they choose. As people with dementia often display agitated behaviours during mid to late afternoon ${ }^{13}$ all sessions will be conducted in the afternoon and in a naturalistic environment (ie, wherever the participants are located at the time of their allocated session, including bedroom, dining area or communal area).

Three members of the team and the project manager will assess adherence to the trial Intervention through random examination of the video observations at regular intervals throughout the trial.

\section{Usual Care}

Participants at sites allocated to the UC treatment group will receive usual care.
A 10-week intervention will allow both short- (5 weeks) and long-term follow-up (10 weeks). We will also conduct a follow-up at 15 weeks, to examine long-term sustainability following withdrawal of the intervention.

\section{Outcomes}

The trial has three primary outcomes of interest: Agitation as measured by the Cohen-Mansfield Agitation Inventory-Short Form (CMAI-SF; 14 short-item) ${ }^{14}$; mood and engagement as measured by video observations via a small GoPro Hero video camera at weeks 0 (baseline), 1, 5, 10 and 15 (follow-up). To ensure a distinct baseline, the first video observation will be conducted in week 0 before the interventions or usual care commence. Direct observations of participants for PARO and PT treatment groups will occur $15 \mathrm{~min}$ before the intervention and during the $15 \mathrm{~min}$ intervention (5 sessions observed in total; total of $2.5 \mathrm{~h}$ per participant). The UC (Control) group individuals will have the video observations undertaken for a $30 \mathrm{~min}$ period during 1300-1700 (5 sessions as per PARO and PT; total of $2.5 \mathrm{~h}$ per participant).

Secondary outcome measures include: sleep duration, step count measured by Sensewear (SW), change in psychotrophic medication use, change in treatment costs and staff and family perceptions of PARO/PT. The SW armband will be placed on individual participants from each group for a $24 \mathrm{~h}$ period at weeks 0 (baseline) and 15 (follow-up) as well as weeks 5 and 10 on a day when the PARO or PT intervention is being delivered or, for the UC group, during usual care (total of $72 \mathrm{~h}$ per participant). To ensure a distinct baseline, the first SW measurement will be conducted in week 0 before the interventions or usual care commences. As needed (PRN) psychotropic medication use will also be measured by a medication audit. Staff and family perceptions of PARO and PT will be assessed in semistructured interviews within a fortnight of the completion of individual interventions with a convenience sample of 20 family and 20 staff. Demographic information of families and staff will be collected, including age, gender, ethnicity, work category (for staff), relationship to person with dementia, qualifications and employment (for family).

\section{Sample size}

In line with outcomes observed in the pilot study and similar outcomes in other research using an individualised intervention for agitation reduction, ${ }^{15}{ }^{16}$ a total sample size of 345 participants, 115 in each group, will be required to detect a $25 \%$ reduction in agitation level (at a power of $0.90, \alpha=0.05$ ). ${ }^{16}$ The sample size was computed using two steps. Sample size required for a simple randomised complete trial design (RCT) was 75 in each group. This was then multiplied by the design effect to account for the nested structure in a C-RCT. Adjusting to account for clustering based on an Intra Class Correlation (ICC) of 0.07 , we determined that at least 
115 patients would be required in each group if we were to use 10 clusters per group $(n=345)$. Allowing for a potential drop out of $10 \%$, increased the total targeted sample to a recruitment sample of 380 participants. To ensure this sample size, we will involve 35 sites in the study with a target of 12 participants at each site.

\section{Randomisation and allocation concealment}

Facilities will be randomly assigned to the PARO, PT and UC groups. Griffith University Centre for Health Practice Innovation will administer the randomisation process centrally via a secure web-based system.

\section{Blinding}

The Intervention Observer RAs will not be blinded to intervention; hence they will not collect outcome data and will be kept blind to all outcome measurements. Intervention Data Collection RAs will be kept blind to the intention of the study and to the alternative conditions. RAs coding videos will be kept blind to the alternative treatment options, as they will each code one treatment group. Family and participants will be kept blind to the outcome measurements. Participants and family will consent only to participation in the study as a whole, not to participation in a particular treatment group.

\section{Data collection}

The study data collected at each time point are summarised in table 1.

\section{Data management}

To preserve confidentiality, all participants will be allocated a unique study identifier, which will be used on all data collection forms alongside participants' initials. A data manager for each treatment group will undertake data entry. A separate password-protected database for each treatment group will allow selected members of the research team to link the participant and the study treatment group. Access to the final data set will be available

\begin{tabular}{|c|c|c|c|c|c|}
\hline & Baseline & $\begin{array}{l}\text { Week } \\
1\end{array}$ & $\begin{array}{l}\text { Week } \\
5\end{array}$ & $\begin{array}{l}\text { Week } \\
10\end{array}$ & $\begin{array}{l}\text { Week } \\
15\end{array}$ \\
\hline Demographics & $x$ & & & & \\
\hline RUDAS & $x$ & & & & \\
\hline CMAI-SF & $x$ & & & $x$ & $x$ \\
\hline $\begin{array}{l}\text { Medication } \\
\text { Audit }\end{array}$ & $x$ & & $x$ & $x$ & $x$ \\
\hline $\begin{array}{l}\text { Sensewear } \\
\text { Measurements }\end{array}$ & $x$ & & $x$ & $x$ & $x$ \\
\hline $\begin{array}{l}\text { Video } \\
\text { observations }\end{array}$ & $x$ & $x$ & $x$ & $x$ & $x$ \\
\hline Interviews & & & & $x$ & \\
\hline
\end{tabular}

only to the research team. Currently no plans for data sharing have been arranged.

\section{Analysis}

Video data will be analysed using Noldus XT Pocket Observer software. Agitated behaviour will be coded according to the internationally validated instrument CMAI-SF. ${ }^{14}$ A coding manual previously developed in our pilot study ${ }^{11}$ will also be used for engagement and mood states. Coders are assigned to coding video data from one treatment group only. This will ensure that coders are blinded to the other treatment alternatives. Furthermore, coding of the preintervention videos before coding of the intervention videos should eliminate bias. Inter-rater reliability, the degree of concordance between coders, will be set at a minimum of 0.90 and 0.85 for $\kappa$ and ICC coefficients, respectively. ${ }^{17}$ This will be assessed and established during training sessions, after which the Noldus trainer will conduct random checks of inter-rater reliability.

Descriptive statistics will be computed for participant demographics and all outcomes measures. An 'Intention-To-Treat' (ITT) approach will be adopted in which all LTC facilities are analysed according to the treatment group into which they were randomised. However, a per-protocol analysis will also be undertaken as a sensitivity analysis. Where appropriate, multiple imputation will be used to manage missing data. Baseline variations between PARO, PT and UC groups will be examined using $\chi^{2}$ tests for categorical variables, $\mathrm{t}$ tests for the normally distributed continuous variables and Kruksal Wallis tests for continuous variables with skewed distributions.

Cluster and individual level analyses will be carried out to test all hypotheses. Generalised Linear Models (GLM) for cluster level and Generalised Estimation Equations (GEE) and/or Multi-Level Modeling (MLM) for individual level data with adjustment for potential covariates at both cluster and individual levels will be used. As covariate data are proposed for collection at both cluster (ie, LTC environmental data) and individual (ie, participant's demographic data, cognitive status and risk of agitation) levels, the adjustment of these factors, if significantly different at baseline between PARO, PT and UC groups, will be carried out when using GEE or MLM as appropriate, depending on the distributional and other assumptions that may vary between the outcome measures. The proposed a priori model-fitting approach will identify (A) each outcome measure and the covariates to be considered for inclusion in any modelling approach; and (B) the potential confounding variables that are to be considered for inclusion in the model with the intervention. STATA software program will be used.

Cluster level analysis: Differences in primary outcome measures (ie, agitation, mood and engagement) between PARO, PT and UC groups, at both short-term and long-term, will be reported. Secondary outcome 
measures (ie, sleep duration, step count and PRN psychotropic medication use) will also be compared between PARO, PT and UC groups. Estimates will be adjusted using GLM for any significant cluster level covariates.

Individual level analysis of the outcome measures when comparing between PARO, PT and UC groups will primarily account for the intracluster correlation (ICC), to increase the statistical power of the analysis. Analyses will include statistical tests, GEE or MLM to account for the clustering effect to avoid spuriously low $\mathrm{p}$ values, overly narrow CIs and over-emphasising the impact of the intervention.

A thematic analysis of the qualitative interviews will explore family and staff perceptions of using PARO and PT in LTC. No interim analyses are planned for this trial.

\section{Comparative cost analysis}

A comparative cost analysis will be undertaken to estimate the costs and cost offsets of the PARO and PT intervention compared with UC. The analysis will take a healthcare provider perspective. The duration of the trial ( 15 weeks) will be used as the time horizon for the within trial analysis; this will be extrapolated to 12 months. Twelve months is consistent with the warranty period of use for PARO. Resource use including the acquisition cost of PARO and additional staff and monitoring costs will be measured during the C-RCT. A weighted cost per day per patient of the intervention will be calculated based on the expected life span of PARO. Cost offsets due to reduction in use of psychotropic medications will be calculated based on the Dispensed Price per Maximum Quantity. Reduction in adverse events such as falls and mortality expected by the reduction in medication usage will be modeled from previous research. ${ }^{18} 19$

\section{TRIAL MANAGEMENT}

Day-to-day running of the trial will be overseen by a team, comprised of the Chief Investigators, Project Manager, Cluster Leaders, and Statistican. Regular team meetings will take place for the duration of the study. The team will have responsibility for ensuring the compliance and progress of the study in relation to regulatory, administrative, industry and safety issues.

\section{Adverse event reporting}

All adverse events will be recorded and reported to the Human Research Ethics Committee, LTC administration and families.

\section{Ethics and dissemination}

After randomisation of sites, potential participants and/ or proxies (if residents are unable to provide informed consent) will be approached by the facility manager and subsequently a project team member for written informed consent to participate in a study exploring robotic animals. They will be informed that participants will receive one of three interventions and that consent is to receive the intervention to which their facility has been assigned and to undergo outcome measures. They cannot consent to receive a particular type of intervention. Where necessary, approval was also sought from facility ethics committees.

Participants will have the right to withdraw from the trial at any time for any reason, without giving a reason. Participants or their representative who no longer wish to participate in the trial may choose to either withdraw completely, or to withdraw from the study intervention, but continue to provide follow-up data. In such a situation, participants or their proxy will be asked if the data collected up to the point of withdrawal may be retained.

\section{Dissemination}

Findings will be presented at local and international conference meetings and published in peer-reviewed journals. A lay summary of the findings will be made available to participants and representatives, and to each facility. If PARO is shown to have an effect, the CIs will develop guidelines to facilitate the use of PARO in LTC facilities.

\section{Trial status}

The trial is currently in data collection. The first of the facilities were recruited on 24th March 2014, facility randomisation took place on 29 May 2014 and participant recruitment started 14 June 2014.

\section{Author affiliations}

${ }^{1}$ Menzies Health Institute Queensland, Griffith University, Brisbane, Queensland, Australia

${ }^{2}$ Centre for Health Practice Innovation, Griffith University, Brisbane, Queensland, Australia

${ }^{3}$ Dementia Collaborative Research Centre, Queensland University of Technology, Brisbane, Queensland, Australia

${ }^{4}$ School of Psychiatry and Dementia Collaborative Research Centre, University of NSW, Sydney, Australia

${ }^{5}$ Behavioural Basis of Health, Griffith University, Brisbane, Queensland, Australia

${ }^{6}$ Faculty of Medicine, Kuwait University, Kuwait City, Kuwait

${ }^{7}$ Centre for Applied Health Economics, Griffith University, Brisbane,

Queensland, Australia

\section{Twitter Follow Wendy Moyle at @wendymoyle2}

Contributors WM had the original idea for the pilot trial, which was followed by this trial. WM, EB, LT, CJ, SO'D developed the trial design. CM developed the comparative cost analysis plan. LT, CJ developed the quantitative data analysis plan and WM developed the qualitative analysis plan. All authors contributed to further development of the protocol including additional amendments and obtained funding. WM wrote this manuscript, SO'D and CJ reviewed the manuscript, and all authors reviewed the final version.

Funding This work was supported by a NHMRC Project Grant APP1065320, 2014-2017.

Competing interests None declared. 
Ethics approval This trial has been granted ethical approval by Griffith University Human Research Committee (NRS/03/14/HREC).

Provenance and peer review Not commissioned research. Application was peer-reviewed for ethical and funding approval prior to submission.

Open Access This is an Open Access article distributed in accordance with the Creative Commons Attribution Non Commercial (CC BY-NC 4.0) license, which permits others to distribute, remix, adapt, build upon this work noncommercially, and license their derivative works on different terms, provided the original work is properly cited and the use is non-commercial. See: http:// creativecommons.org/licenses/by-nc/4.0/

\section{REFERENCES}

1. Australian Institute of Health and Welfare. Dementia in Australia. Canberra, AlHW, 2012. Cat no. AGE 70.

2. World Health Organisation. Dementia: a public health priority. Switzerland: World Health Organisation, 2012.

3. Yeager CA, Hyer L. Apathy in dementia: relations with depression, functional competence, and quality of life. Psycho Rep 2008:102:718-22

4. Brodaty H, Draper B, Low LF. Nursing home staff attitudes towards residents with dementia: strain and satisfaction with work. J Adv Nurs 2003;44:583-90.

5. Astrom S, Nilsson M, Norberg A, et al. Staff burnout in dementia care: relations to empathy and attitudes. Int J Nurs Stud 1991;28:65-75.

6. Maher AR, Maglione M, Bagley S, et al. Efficacy and comparative effectiveness of atypical antipsychotic medications for off-label uses in adults: a systematic review and meta-analysis. JAMA 2011;306:1359-69.

7. Libin A, Cohen-Mansfield J. Therapeutic robocat for nursing home residents with dementia: preliminary inquiry. Am J Alzheimer Dis Other Demen 2004;19:111-16.

8. Kramer S, Friedman E, Bernstein P. Comparison of the effect of human interaction, animal-assisted therapy, and AIBO-assisted therapy on long-term care residents with dementia. Anthrozoös 2009;22:43-57.

9. Tamura T, Yonemitsu S, Akiko I, et al. Is an entertainment robot useful in the care of elderly people with severe dementia? J Gerontol A Biol Med Sci 2004;59:83-5.

10. Wada K, Shibata T. Living with seal robots-its sociopsychological and physiological influences on the elderly at a care house. IEEE Trans Robot 2007;23:972-80.

11. Moyle W, Cooke M, Beattie E, et al. Exploring the effect of companion robots on emotions in older people with dementia: a pilot RCT. J Gerontol Nurs 2013;39:46-53.

12. Rowland JT, Basic D, Storey JE, et al. The Rowland Universal Dementia Assessment Scale (RUDAS) and the Folstein MMSE in a Multicultural Cohort of Elderly Persons. Int Psychogeriatr 2006;18:111-20.

13. Khachiyants N, Trinkle D, Son SJ, et al. Sundown syndrome in persons with dementia: an update. Psychiatry Investig 2011;8:275-87.

14. Cohen-Mansfield J. Conceptualization of agitation: results based on the Cohen-Mansfield Agitation Inventory and the Agitation Behaviour Mapping Instrument. Int Psychogeriatr 1996;8:309-15.

15. Koss E, Weiner M, Ernesto C, et al. Assessing patterns of agitation in Alzheimer's disease patients with the Cohen-Mansfield Agitation Inventory. Alzheimer Dis Assoc Disord 1997;11(Suppl. 2): S45-50.

16. Chenoweth L, King MT, Jeon YH, et al. Caring for Aged Dementia Care Resident Study (CADRES) of person-centred care, dementia-care mapping, and usual care in dementia: a cluster-randomised trial. Lancet Neurol 2009;8:317-25.

17. Hinze JL. Pass 2005 user's guide. Kaysville, UT: Number Cruncher Statistical Systems, 2004

18. Campbell AJ, Robertson MC, Gardner MM, et al. Psychotropic medication withdrawal and a home-based exercise program to prevent falls: a randomized, controlled trial. J Am Geriatr Soc 1999;47:850-3.

19. Ballard C, Hanney ML, Theodoulou M, et al. The dementia antipsychotic withdrawal trial (DART-AD): long-term follow-up of a randomised placebo-controlled trial. Lancet Neurol 2009;8:151-7. 